\title{
Identification and characterization of conserved miRNAs with its targets mRNA in Trichinella Spiralis
}

\author{
Dyavegowda Padmashree \& Narayanaswamy Ramachandraswamy* \\ Department of Biochemistry, Central College Campus, Bangalore University, Bangalore, Karnataka, India; Narayanaswamy \\ Ramachandraswamy - E-mail: drnrswamy@bub.ernet.in; Corresponding author; Telephone number: +91 08022961335
}

Received June 16, 2016; Revised July 10, 2016; Accepted July 11, 2016; Published August 15, 2016

\begin{abstract}
:
microRNAs (Small regulatory non-coding RNAs) have an important role in gene regulation and evolutionarily conserved molecules. Trichinella spiralis infect majority of species. Therefore, it is of interest to identify conserved miRNAs and their targets using sequences from EST, GSS and full length nucleotides obtained from NCBI against previously reported worm miRNAs. We identify 11 novel miRNAs in $T$. spiralis by using bioinformatics-homology based search. In addition, we predicted target mRNA genes form complementary base pair in seed region of miRNAs. Further, gene annotation using Uniprot shows that these target genes of miRNAs are involved in various metabolism, enzymatic activity and constituents of membrane components.
\end{abstract}

Keywords: miRNA, computational, T. spiralis, prediction, annotation

\section{Background:}

MicroRNAs (miRNAs) are endogenous small, single stranded, non-coding RNA ( 19-25 nucleotides) molecules that regulate gene expression at post-transcriptional level either by mRNA cleavage or repressing translation [1, 2]. Larger number of miRNAs have been identified and characterized in various organisms such as animals, plants, viruses and in parasites [3-5]. miRNA sequences are highly conserved across the species [6, 7]. Therefore, it is of interest to identify and characterize conserved miRNA in $T$. spiralis by using computational analysis.

Nematode of the genus Trichinella are zoonotic parasites which are distributed Worldwide [8,9]. Different species in the genus Trichinella have been identified. Trichinella spiralis (T. spiralis) is most prevalent and pathogenic disease causing nematode in human. It causes food-borne diseases called trichinellosis which infect majority of species involving human [10]. Human trichinellosis has been documented in 55 countries, hence considered as an emerging or re-emerging diseases [11-13]. Infection occurs by consumption of raw or undercooked meat contaminated with infective muscle larvae of the parasite [14, 15]. After ingestion muscle larvae, releases from their capsules and gets activated into the intestinal infective larvae and then ISSN 0973-2063 (online) 0973-8894 (print) penetrate into the host intestinal epithelium where they molt and mature to adult worm [16]. The female worm reproduce the new born larvae, they are passed into tissue, enter the lymphatic system and travel through the bloodstream into the muscle fibre, once the worms reach the muscular tissue it gets induced into encapsulated nurse cell $[\mathbf{1 7}, \mathbf{1 8}]$ which can survive in host for years. They then wait for a new host infection, thereby the new generation begins. However no miRNA has been reported in miRBase for this genus which provided new opportunity for predicting conserved novel miRNAs and their putative target genes. Identification of miRNAs and the function of target genes in $T$. spiralis may provide the idea to know the mechanism of infection and their metabolic processes.

\section{Methodology:}

Collection of sequences and reference miRNAs set

T. spiralis EST (expressed sequence tag), GSS (genome survey sequence) and nucleotide sequences were retrieved from NCBI (http://www.ncbi.nlm.nih.gov/), used for miRNA prediction. A total of 25,368 sequences were deposited in EST database (dbEST) (http://www.ncbi.nlm.nih.gov/dbEST/), 1 GSS and 35,787 nucleotides respectively. To identify the potential conserved miRNA in T. spiralis, previously known mature miRNA 
sequences belonging to the phylum Platyhelminthes, Nemertea, Annelida and Nematoda were retrieved from miRBase database Release 21 (http://www.mirbase.org/cgi-bin/browse.pl) [19]. These mature miRNA sequences were used as reference miRNAs set to search the conserved miRNAs in T. spiralis.

\section{Computational tools}

The freely available BioEdit software [20] is used for local alignment tool BlastN search, used to predict conserved miRNA in T. spiralis using EST, GSS and nucleotides. The sequences were retrieved from NCBI and saved in FASTA format. Commercial available Geneious software version 7.1.5 were used for pairwise and multiple alignments with default parameter, in addition, it contain RNAfold program [21] to predict secondary structure of pre-miRNAs.

\section{Nomenclature of miRNAs}

The predicted miRNA in T. spiralis were named according with miRBase [22]. The mature miRNA sequence are designed with ' $\mathrm{miR}$ ', and precursor hairpin are labelled as 'mir' with the prefix in three letter 'tsp' for Trichinella spiralis, first letter from genus and first two letter from species.

\begin{tabular}{|c|c|c|c|c|c|c|}
\hline tsp-miRNAs & Length & Mature miRNA sequences & $(\mathrm{G}+\mathrm{C}) \%$ & MFE (kcal/mol) & AMFE & MFEI \\
\hline 9 & 120 & UCUUUGGUUAUCUAGCUGUAUGA & 34.8 & -45.7 & -38.9 & 1.0 \\
\hline 34 & 71 & UGGCAGUGUAAUUAGCUGGUUG & 45.1 & -32.7 & -46.0 & 1.0 \\
\hline 72 & 120 & AGGCAAGAUGUUGGCAUAGCUGA & 51.7 & -45.9 & -38.2 & 0.7 \\
\hline 79 & 120 & AUAAAGCUAGGUUACCAAAGCUA & 44.2 & -43.6 & -36.3 & 0.8 \\
\hline 86 & 120 & UAAGUGAAUGCUUUGCCACAGACU & 44.2 & -43.6 & -36.3 & 0.8 \\
\hline 100 & 84 & AACCCGUAGAUCCGAACUUGUGUU & 54.8 & -29.9 & -35.6 & 0.6 \\
\hline 124 & 123 & UAAGGCACGCGGUGAAUGCCAA & 51.2 & -47.2 & -38.3 & 0.7 \\
\hline 125 & 91 & UCACAAGUUUGGGUCUCAGGGA & 48.4 & -28.3 & -31.1 & 0.7 \\
\hline 153 & 108 & CAUCACUUUUGUGACUAUGCAA & 44.4 & -35.2 & -32.6 & 0.7 \\
\hline 252 & 120 & CUAAGUAGUAGUGCCGCAGGUCA & 49.2 & -46.5 & -38.8 & 0.8 \\
\hline 263 & 82 & GCCGUGAAUUCAUCCAGUGCCAUU & 45.1 & -29.7 & -36.21 & 0.8 \\
\hline
\end{tabular}

Table 1: Predicted characterized features of precursor miRNA in T. spiralis are given.

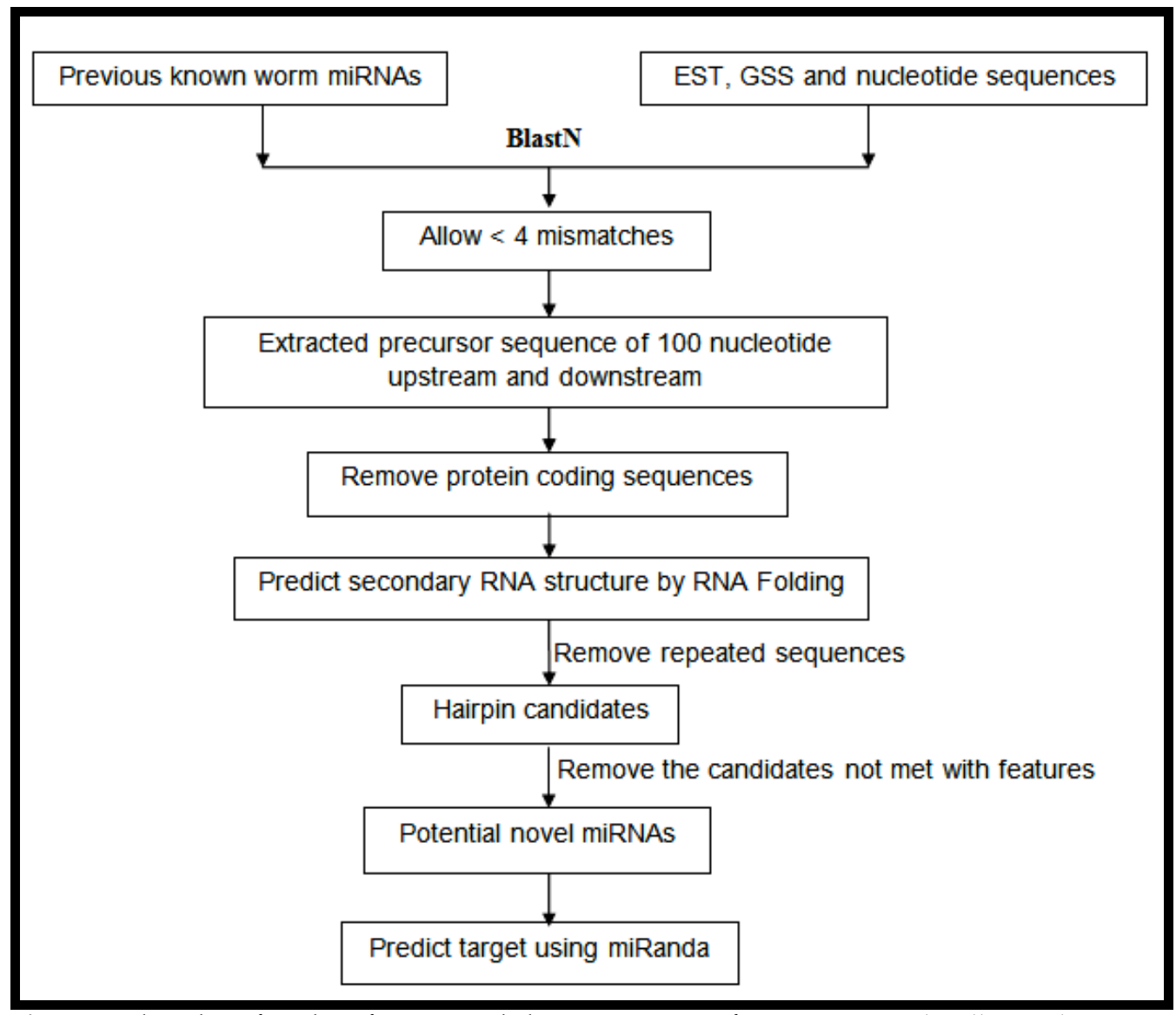

Figure 1: Flowchart for identification and characterization of miRNA in Trichinella spiralis.

ISSN 0973-2063 (online) 0973-8894 (print) 


\section{BIOINFORMATION}

\section{Discovery at the interf ace of physical and biological sciences}

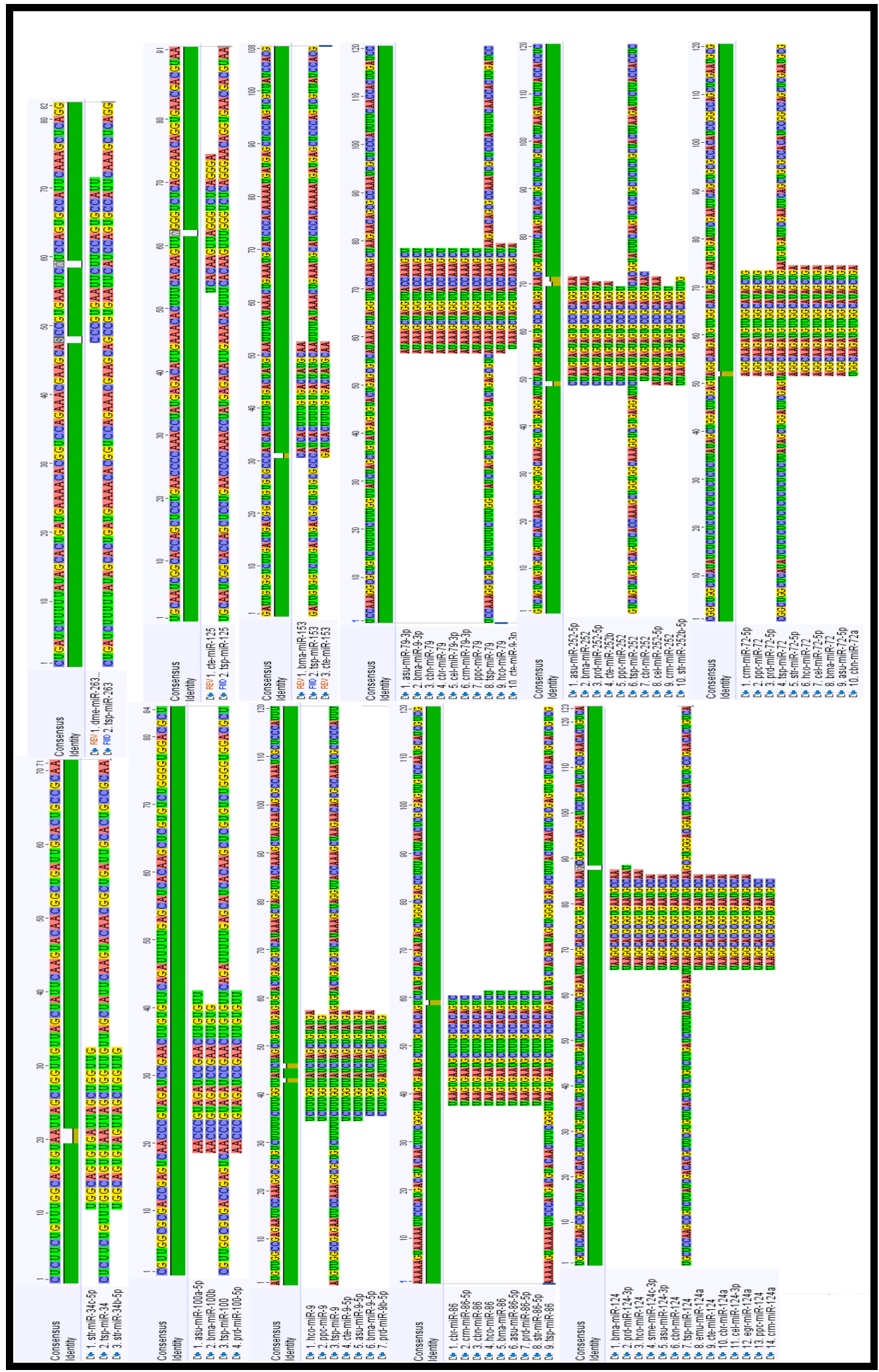

ISSN 0973-2063 (online) 0973-8894 (print)

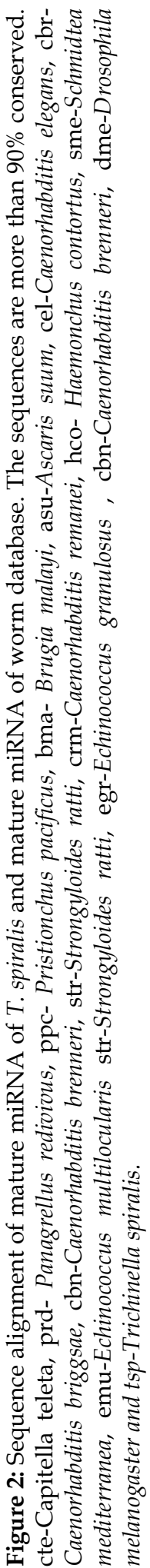

Open access 


\section{BIOINFORMATION}

\section{Discovery at the interface of physical and biological sciences}

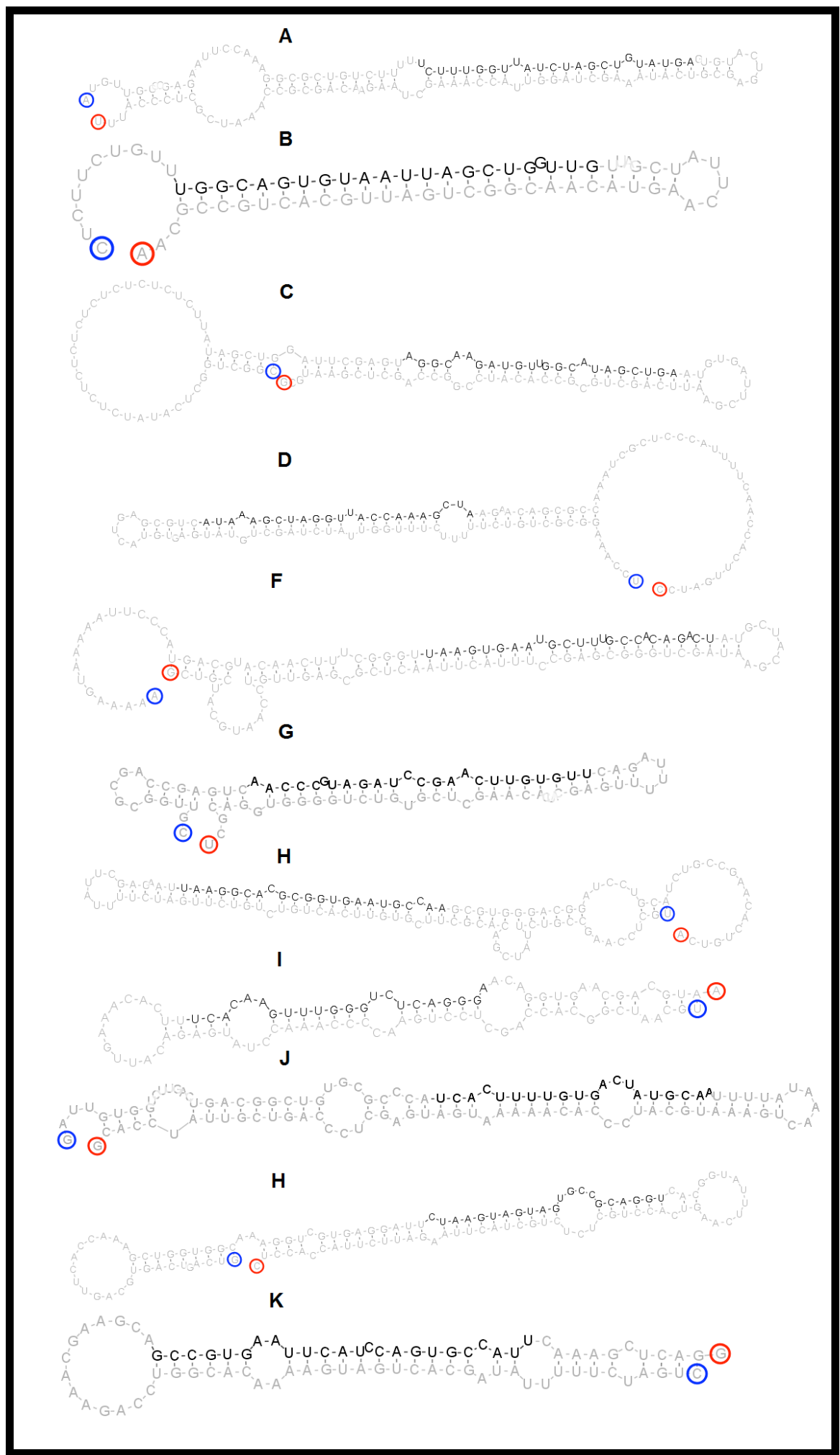

Figure 3: Predicted hairpin secondary structures of newly identified miRNAs in T. spiralis. The mature miRNA sequences are highlight in dark black colour. miR-9; B. miR-34; C. miR-72; D. miR-79; E. miR-86; F. miR-100; G. miR-124; H. miR-125; I. miR-153;

J. miR-252 \& K. miR-263

ISSN 0973-2063 (online) 0973-8894 (print)

\section{BIOMEDICAL}




\section{Open access}

Identification and prediction of precursor microRNAs

Procedure for identifying potential conserved novel miRNAs is summarized in Figure 1. The following steps were considered: local nucleotide BLAST were run with the reference miRNAs set sequences searched against $T$. spiralis sequences at low e-value threshold using BioEdit software. The results of BLASTN were further analyzed by applying two conditions: 1) at least 19-24 nucleotides length were assumed between the predicted mature miRNAs, more than $90 \%$ identity between the sequences. 2) Mismatches between the sequences with 0-4 nucleotide are allowed with reference set of worm mature miRNAs.

The precursor sequences were searched at 50 nucleotides upstream and downstream from the location of mature miRNAs. The following conditions are considered to predict miRNA sequences according to Zhang [23]. 1) The minimum length of pre-miRNA to be 60 nucleotides. 2) The precursor miRNA sequences can fold into an appropriate hairpin secondary structure, the mature miRNA sequence site may be in any one arm of hairpin structure. 3) Not more than 6 mismatches between predicted mature miRNA and the opposite miRNA* sequence in the hairpin structure. 4) No breakage or loop in mature miRNA and miRNA* sequences. 5) Predicted secondary structures had retained minimum free energy (MFE) ( $\leq-18 \mathrm{kcal} / \mathrm{mol})$. 6) $30-70 \%$ of $\mathrm{G}+\mathrm{C}$ content.

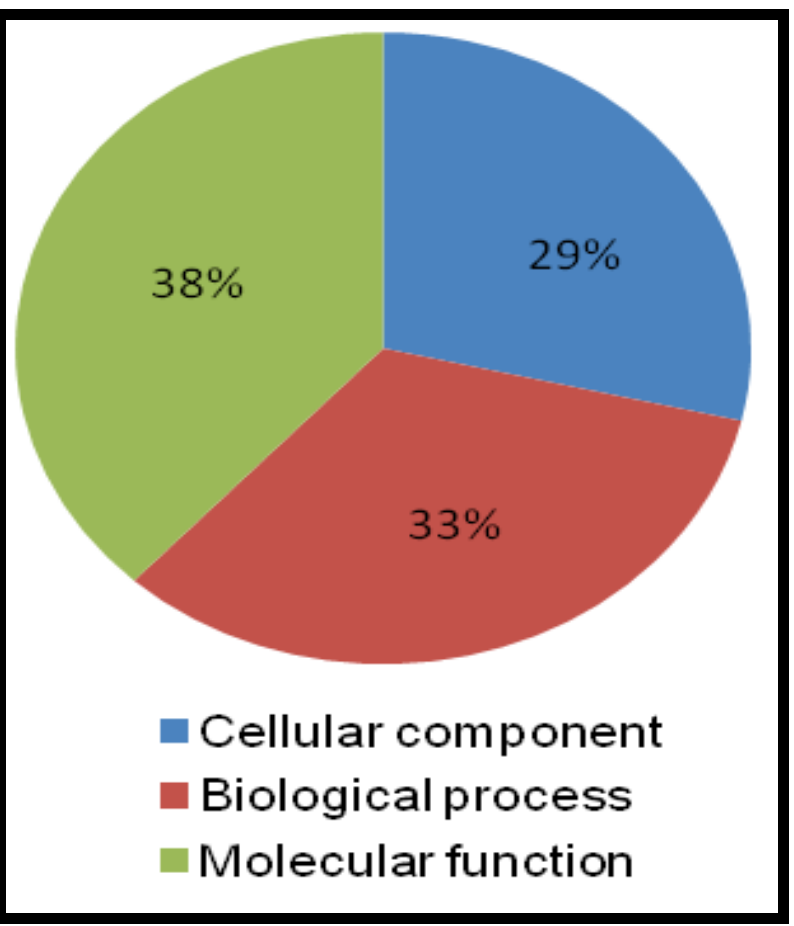

Figure 4: Pie chart showing Functional categories (cellular component, biological process and molecular function) of predicted target genes of novel miRNAs in T. spiralis.

\section{miRNAs target prediction and annotation}

Totally 16,589 gene sequences are available in NCBI for T. spiralis. To search the targets we prefer miRanda software version $3.3 \mathrm{a}$
[24], released 2010 available at http://www.microrna.org /miranda. The program used thermodynamics and dynamic programming alignments, along with statistical parameters were followed in these studies with the MFE threshold $-20 \mathrm{kcal} / \mathrm{mol}$, Score Threshold 120, Scaling Parameter 4 for complementary nucleotide match score. Outputs were subsequently screened out to reduce false positive results. In addition, function of predicted target genes are allowed to understand regulatory network in the cellular, biological and molecular processes using gene annotation Uniprot database (www.uniprot.org).

\section{Results and Discussion:}

In silico prediction of miRNAs in T. spiralis

Different computational approaches were used to identify miRNA in both animal and plants [25-27]. In the present study, we identify the conserved miRNAs in T. spiralis using EST, GSS and nucleotides (DNA and RNA sequences) by comparative genomic approach. The collected sequences from these sources were retrieved and saved in FASTA format. In BioEdit software we created local blast for nucleotide database as reference miRNAs set which contains all known worm mature miRNA sequences retrieved from miRBase, then BlastN program were performed against the $T$. spiralis sequences with default parameter and low e-value threshold. The resulted output were filtered by applying two conditions 1) at least 18-24 nucleotides length were assumed between the predicted mature miRNAs, more than $90 \%$ identity between the sequences. 2) Mismatches between the sequences with 0-4 nucleotides are allowed with reference sets of worm mature miRNAs. Figure 2 shows the high degree of sequence similarity between the predicted mature miRNA sequences and previously known miRNAs. Gap in alignment is due to insertion or deletion of the nucleotides. Further, the precursor sequences were searched at 50 nucleotides upstream and downstream from the location of mature miRNA. Since miRNA are non-coding nucleotides, we performed BLASTX 2.2.30 search against non-redundant (NR) protein databases in order to discard the protein coding miRNA candidates. The remaining precursor miRNA candidates were analysed to predict secondary structure by using RNAfold. Figure 3 shows precursor miRNA and mature sequences in stem loop structures. All the mature miRNA were found in the stem portion of hairpin structures containing less than 6 mismatches in the opposite arm without break or loop inside the mature sequences. Mature sequence resides either in $3^{\prime}$ arm or in $5^{\prime}$ arm of the secondary structures. In our studies we considered from 60-120 nucleotides and 19 to 24 nucleotides of precursor miRNAs and mature miRNA sequences respectively. Within the genome, repeated sequences were removed, finally we identified 11 potential novel miRNAs namely tsp-miRNA-9, 34, 72, 79, 86, 100, 124, 125, 153, 252 and 263 respectively.

\section{Characterization of $T$. spiralis miRNA}

In our study, we could able to identify some of the features of precursor miRNA, such as MFE, have a predominant role to determine the secondary structure of nucleic acids. Lower the MFE, higher the stability of the structure [28]. Adjusted minimal 
free energy (AMFE), is calculated by (MEF/Length of RNA sequence) ${ }^{*} 100$, minimal free energy index (MFEI) calculated by AMFE/ $(\mathrm{G}+\mathrm{C}) \%$. All this features are calculated for each miRNA candidates. MFEI is an important criteria to distinguish miRNA from other types of RNA (coding and non-coding) [23]. In our studies MFE range from -28.3 to $-47.2 \mathrm{kcal} / \mathrm{mol}$ respectively, GC content $(30-70 \%)$ and MFEI ranges from 0.6 to 1.0 Table 1.

\section{miRNA Target genes and their function}

Generally, miRNAs regulate gene expression through translational repression or mRNA cleavage. To understand the target genes of predicted miRNA candidates in T. spiralis, we used miRanda software with the above mentioned criteria. miRNA have potential target site are most commonly found in the 3'UTR of mRNA, which search for the sequence for complementary base site recognised 2-8 nucleotides (seed region) from the 5 'end of the mature miRNA. After filtering certain criteria 843 targets were obtained for 11 novel miRNA candidates.

Further the functional information of each miRNA target genes were search using Uniprot database. Gene annotations were functionally categorized into three parts: cellular component, biological process and molecular functions. In our analysis around $38 \%$ are contribute for molecular function, 33\% and $29 \%$ responsible in biological process and cellular component respectively Figure 4. The target genes in the biological category are highly involved in various metabolism and signal transduction, in the cellular process mainly the component of the membranes followed by nucleus and in the molecular process highest percentage in enzymatic activity, nucleic acid and ion binding. The predicted miRNA candidates target genes were found in various regulatory roles during growth and developments. Our studies help us to understand important regulatory network and roles of miRNAs in T. spiralis.

\section{Conclusion:}

T. spiralis is food borne zoonotic nematode causative agent of trichinellosis in Human. Our study is the first attempt to identify and characterize conserved novel miRNAs with its target mRNA in T. spiralis. In this report we identify 11 novel miRNA candidates which were evolutionarily conserved with previously known miRNAs register in miRBase. Analysis of miRNA targets within the genome of $T$. spiralis, help to understand the regulatory function of each miRNA candidates. In addition, gene annotation analysis shows that most of the target transcripts are involved in various metabolism, enzymatic activity and constituents of membrane components. Overall this helps to understand conserved miRNAs in evolution, genome organization and gene regulatory networks in T. spiralis. The predicted result from computational approach would be the next step in future to guide experimental methods to design, which would through more light into the pathogenesis related control, in better understanding of prevention of infection.

\section{References:}

[1] Bartel DP, Cell. 2004 116:281. [PMID: 14744438]

[2] Pillai RS et al. Trends in cell biolog.y 2007 17:118. [PMID: 17197185]

[3] Zhang B et al. J Cell Physiol. 2007 210:279. [PMID: 17096367]

[4] Grundhoff A et al. RNA. 2006 12:733. [PMID: 16540699]

[5] Xue $X$ et al. PLoS ONE 2008 3:e4034. [PMID: 19107204]

[6] Wheeler BM et al. Evol Dev. 2009 11:50. [PMID: 19196333]

[7] Weber MJ, FEBS J. 2005 272:59. [PMID: 15634332]

[8] Dupouy-Camet J, Vet Parasitol. 2009 159:194. [PMID: 19054620]

[9] Dupouy-Camet J, Vet Parasitol. 2000 93:191. [PMID: 11099837]

[10] Pozio E, Vet Parasitol. 2007 149:3. [PMID: 17689195]

[11] Murrell KD et al. Emerg Infect Dis. 2011 17:2194. [ PMID: 22172230]

[12] Cui J et al. Vet Parasitol. 2013a 194:133. [PMID: 23422779]

[13] Dupouy-Camet J, Vet Parasitol 2009 159:194. [PMID: 19054620]

[14] Campbell W C et al. Ed Istituto Superior di Sanita Press Rome, Italy. 1993.

[15] Krol-Pakulska E et al. Przegl Epidemiol. 2008; 62:553. [PMID: 19108518]

[16] Gagliardo LF et al. Infect Immun. 2002; 70:1853. [ PMID: 11895947]

[17] Polvere RI et al. Exp Parasitol. 1997; 86:191. [PMID: 9225769]

[18] Despommier DD, Parasitol. Today 1998; 14:318. [PMID: 17040798]

[19] Griffiths-Jones S et al. Nucleic Acids Res. 2008 36: D154. [PMID: 17991681]

[20] Hall TA, Nucleic Acids Symposium Series. 1999 41: 95.

[21] Hofacker IL, Nucleic Acids Res. 2003 31: 3429. [PMID: 12824340]

[22] Griffiths-Jones S et al. Nucleic Acids Res. 2006 34: D140. [PMID: 16381832]

[23] Zhang BH et al. Cell Mol Life Sci. 2006 63: 246. [PMID: 16395542]

[24] Enright AJ et al. Genome Biol. 2003 5:R1. [PMID: 14709173]

[25] Lai EC et al. Genome Biol. 2003 4: R42. [PMID: 12844358]

[26] Jones-Rhoades MW \& Bartel DP, Mol Cell. 2004 14: 787 [PMID: 15200956]

[27] Brown JR \& Sanseau P, Drug Discov Today. 2005 10: 595 [PMID: 15837603]

[28] Bonnet E et al. Bioinformatics. 2004 20: 2911. [PMID: 15217813]

Edited by P Kangueane

Citation: Padmashree \& Ramachandraswamy, Bioinformation 2016 12(5) 279-284. License statement: This is an Open Access article which permits unrestricted use, distribution, and reproduction in any medium, provided the original work is properly credited. This is distributed under the terms of the Creative Commons Attribution License. 